\title{
Variations Between Perceptions of Interpersonal Distance in Virtual Environments for Autism
}

Ciera Crowell

University Pompeu Fabra

Department of Information and

Communication Technologies

Carrer Roc Boronat 138

08018 Barcelona

ciera.crowell@upf.edu

Narcis Pares

University Pompeu Fabra

Department of Information and

Communication Technologies

Carrer Roc Boronat 138

08018 Barcelona

narcis.pares@upf.edu
Permission to make digital or hard copies of part or all of this work for personal or classroom use is granted without fee provided that copies are not made or distributed for profit or commercial advantage and that copies bear this notice and the full citation on the first page. Copyrights for third-party components of this work must be honored. For all other uses, contact the owner/author(s). CHI PLAY'18 Extended Abstracts, Oct. 2831, 2018, Melbourne, Australia. Copyright is held by the author/owner(s). ACM ISBN 978-1-4503-5968-9/18/10. https://doi.org/10.1145/3270316.3271537

\begin{abstract}
Interpersonal distance is defined as the area which we choose to keep between ourselves and others, revealed through observation and cultural components. Although previous studies have suggested the possibility of alterations in perception of interpersonal distance in children with Autism Spectrum Disorder, it remains unknown whether these differences exist in relation to characters in a virtual environment. As many social-skills interventions for autism rely upon virtual characters to teach social behaviors, this research is key in understanding how to configure the interpersonal distance of virtual characters to an adequate level to effectively foster computerized social-skills training. We have carried out controlled trials with children with autism to identify variations in preferences from the typically developed population with both a human partner and a virtual character. The contributions of this research are twofold: first, to support existing literature in identifying differences in personal space preferences between children with autism and typically developing children; and second, to understand whether these differences carry over into the context of virtual environments.
\end{abstract}

\section{Author Keywords}

Autism Spectrum Disorder, ASD, Interpersonal distance, Proxemics, Virtual environments 


\section{Introduction}

It is a common sentiment to ponder how it would be to walk in someone else's shoes for a day. Similarly, in the study of human-computer interaction, designers try to understand the use of interactive systems through the eyes of our target user group. However, challenges arise when approximating the desired use of interactive systems for target users who have alterations in cognitive functioning. This work aims to identify whether these alterations might result in variations in the perception of interpersonal distance, ultimately towards the design of virtual environments which accommodate interpersonal space differences.

Individuals with Autism Spectrum Disorder (ASD) perceive the world in a unique manner which reflects the nature of their condition. The field of research which focuses on Autism has identified three main characteristics which contribute to this particular perception:

communication challenges, a tendency to engage in repetitive behaviors, and social-interaction difficulties [1]. The differences which pertain to this condition shed light on the manner in which these individuals may interact with information and communication technologies, an area of heightened interest for children with ASD. With respect to social-interaction difficulties, much research has focused on creating collaborative scenarios for practicing and strengthening social capabilities in these children [9]. However, these projects have not considered how interpersonal distance specific to ASD could be utilized as a way of fostering social behaviors between users. As proxemics theory has been implemented in interactive systems as a way to understand device to people relations [2] and social interaction between typically developing users [10], we believe this work could be valuable in the design of interactive systems for social intervention in children with ASD.

Our aim with this research study is to identify particularities in the perception of personal space with virtual agents for a specific user group, namely those with Autism Spectrum Disorders. Ultimately our aim is to contribute to the creation of interactive systems through a better understanding of personal space preferences for a target user group, in real life and virtual scenarios.

\section{Background}

Interpersonal distance is present in each interaction which we conduct face-to-face with others. Although largely subconscious, these distances become evident as we orient ourselves towards others in social situations, typically in four distinct zones: intimate space, personal space, social space, and public space. Cultural differences in interpersonal space preferences have been observed through the study of proxemics [7], along with differences related to age, sex, personal traits, and nature of the relationship.

In an experimental setting, interpersonal distance is often tested through the use of a stop-distance test. This simple but widely-recognized test involves one subject remaining stationary at a designated point as an

interlocutor gradually approaches by walking head-on [8]. When the subject feels as if the interlocutor has reached the edge of their comfort threshold, they make a signal, often by saying "stop." This test has been used with children with ASD to detect personal space preferences before and after socializing with an adult confederate [6]. When compared to typically developing children, the children with ASD preferred a slightly greater distance between themselves and the adult confederate. 
Personal space preference may also be detected through naturalistic observation. In an experiment by Rogers and Fine [12], a child with ASD and a child with symbiotic psychosis were observed for preferences in personal space during play therapy sessions with a therapist.

Observations revealed that the child with ASD preferred a significantly greater distance between himself and the therapist than the symbiotic child.

\section{Proxemics in computer based systems}

An early mention of possible inconsistencies with proxemic perception in children with ASD came when Parsons created and tested the Virtual Cafe system, an intervention tool for social skills in which children experienced simulations of real-life scenarios such as finding a seat on a bus or navigating through a cafeteria [11]. In this system, it was observed that the children with ASD bumped into virtual characters representing people, a behavior which differed from the typically developed users. From this arose the question of whether children with ASD might have impairments in personal space preferences, and particularly in relation to virtual characters.

In addition, Welch et al. detected the response of children with ASD to various measures of social distance and eye gaze of a humanoid avatar in a virtual environment [13]. Children with ASD seemed to show unusual reactions to the avatars when standing at an invasive distance or using increased amounts of eye contact, to which they sometimes reacted by leaning far back or looking away from the monitor. Boyd, et al. explored the use of VR devices which present a visible display of nonverbal social cues in vrSocial [4] and ProCom [3], which included a proximity visualization between users.

Proxemics were used to evaluate the reaction of children with ASD during a free play scenario with a humanoid robot [5]. The children showed mixed positive and negative reactions to the robot, given that 7 children spent a significant amount of time interacting with the robot, and 6 children spent the majority of time avoiding it.

As discussed, although children with ASD have been observed to maintain a greater distance between themselves and human counterparts, it is not clear how this tendency carries over into computerized settings which contain virtual agents. This study aims to identify idiosyncrasies in personal space between children with ASD and human partners, and look for the same pattern when presented with a virtual character.

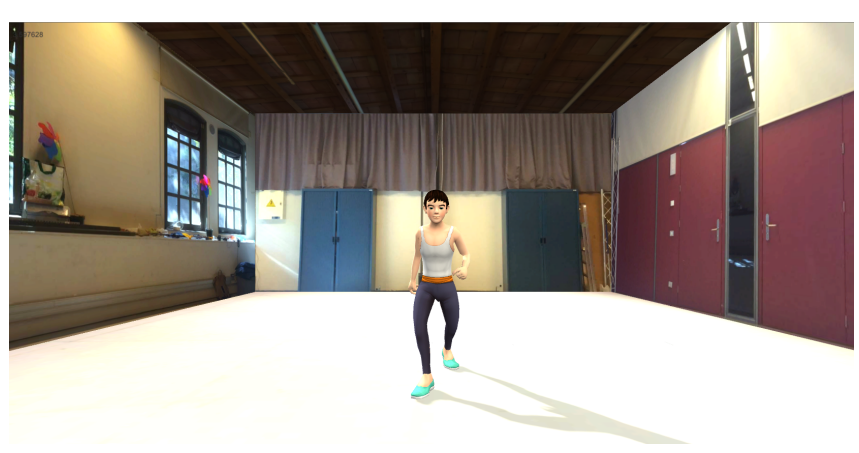

Figure 1: Children indicated preferred personal space towards a life sized virtual character within a laboratory simulation

\section{Methodology}

The experimental protocol consisted of two parts to detect irregularities between preferences towards personal space.

The first scenario included a brief baseline interaction where one user would indicate their preferred comfort distance in relation to another child, who they had not met before the experiment. In the second scenario, the user would indicate their preferred comfort distance in 
relation to a virtual character. We chose these two scenarios as we ultimately wanted to use this information in the development of multiplayer virtual environments, and therefore saw it necessary for the children to show distance preferences in relation to a virtual character and also a potential human playing partner. The two children were then asked to play a 15 minute open ended play activity together, before completing the stop distance test with their partner a final time.

We tested both settings in an interactive laboratory setting with 32 children between the ages of 10-15 (6 female; 26 male). Each pair consisted of one child with autism $(n=16)$ and a typically developing partner $(n=16)$. The observations and subsequent adaptations are outlined in this section.

\section{Human interlocutor configuration}

To evaluate differences in perception of personal space between children with ASD and typically developing children, we used the stop-distance paradigm, where the experimental subject stood behind a marked line as an interlocutor approached them by walking head-on at a relaxed pace. In our case, we used an initial distance of 6 meters which is commonly recognized within the largest proxemic zone (the "public" zone). The subject is typically asked to verbalize when the interlocutor is too close, and the resulting distance between interlocutors is measured. During pilot tests, we saw that it would be necessary to adapt the stop distance test to the specifications of the autistic condition. Most notably, we noticed that children with ASD had difficulties verbalizing when they wanted their partner to stop. Therefore, we gave each child a hand-held button to push instead of saying 'stop.' The children were also told to greet their partner in a friendly way as they approached by performing a hand-waving gesture at the desired stopping point. These measures helped us to record the moment when the interlocutors were asked to stop, without requiring the children to make verbal commands.

\section{Virtual character configuration}

In addition to the stop-distance test with human counterparts, children were also asked to perform a similar exercise with a virtual counterpart. For this test, children were asked to stand in front of a large $(1 \times 2$ meter $)$ projection screen. On the screen was projected a three dimensional virtual environment with a human character standing in the center (see Figure 1). The virtual environment was modeled after the laboratory where we conducted testing, so that the view on the screen would resemble the interior of the testing room from the position of the child during the stop distance test. The camera was set at the average height of a 10 year old child. The virtual character was a young male in plain clothes, scaled to average size within the virtual environment, and placed initially 6 virtual meters back from the camera. When the test began, the child remained stationary as the virtual character walked toward the camera. The children were asked to push their button and wave just when the virtual character breached their comfort distance. At that point, the character assumed a standing position and the computer generated a measurement of the distance between the camera viewing point and the virtual character.

\section{Results}

The children with ASD showed a preference for increased distances between themselves and their human

interlocutors $(\mathrm{M}=156.25 \mathrm{~cm}, \mathrm{SD}=81.11)$ when compared to the typically developing partners $(M=139 \mathrm{~cm}$, $S D=60.20)$, as seen in Figure 2. Children with ASD 
showed a tendency for a much larger distance between themselves and the virtual character than a human partner, as did the typically developing children. Although the results were largely similar, as seen in Figure 3, the group of children with autism showed a slightly lower preference for distance between themselves and the virtua character $(M=268.47 \mathrm{~cm}, \mathrm{SD}=125)$ than did the typically developing children $(\mathrm{M}=276.87 \mathrm{~cm}, \mathrm{SD}=82.11)$.

Interestingly, after a 15 minute interaction with their partner, the children with autism showed a much larger decrease in preference for personal space with their partner $(M=116.93 \mathrm{~cm}, S D=64.41)$ than did the typically developing children $(\mathrm{M}=142.2 \mathrm{~cm}, \mathrm{SD}=74.44)$.
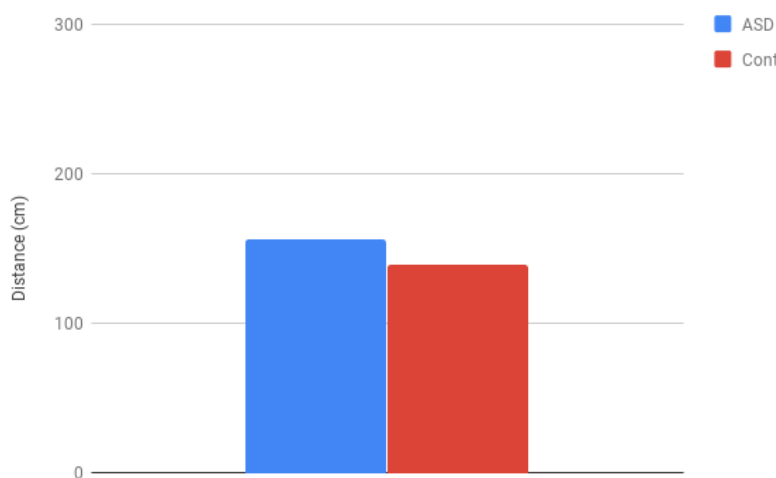

Figure 2: Distance preferences with a child partner

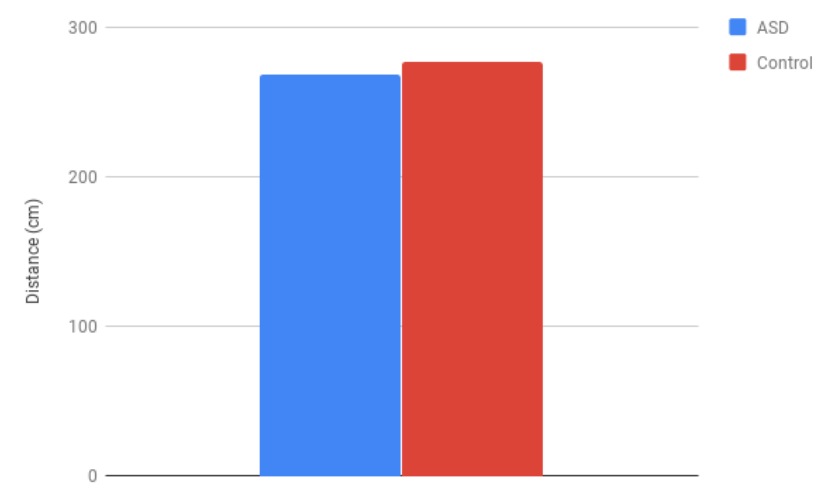

Figure 3: Distance preferences with a virtual character

\section{Discussion and Conclusion}

As shown in previous literature, children with autism preferred a larger personal space between themselves when presented with a child partner, as indicated by the results of the stop distance testing procedure. This pattern was not carried over into the virtual environment setting, as no significant differences existed between populations of children when presented with a virtual character. This calls into question assumptions with regard to previous observations where children with autism showed irregular behaviors when presented with a virtual character at close proximity. In our case, the children with autism were not comfortable with the virtual character in close proximity, but this pattern was shared by the typically developing children. As both groups of children were not comfortable with the virtual agent when it breeched the social space barrier, this indicated that the virtual character might have been perceived as a real being by both children with autism and typically developing children alike. This finding also brings up possibilities for research on why children with ASD might show a stronger aversion to close 
contact with people, rather than with virtual characters, when compared to typically developing populations.

It is important to note that the perception of the virtual counterpart and the human counterpart were not directly comparable, as conditions related to the virtual environment (viewing angle, believability of the virtual agent) meant the two scenarios differed in key ways. Our goal was rather to search for differences between the two populations with regard to the various conditions presented. In addition, this experiment was done with one virtual character, selected for the similarity in appearance to the predominantly male population of individuals with ASD in this study. Future experiments might test the reaction towards various designs of virtual characters, to analyze the effect of factors which traditionally affect personal space preference (age, sex, personal traits).

Future research might strive to reduce these differences and also compare reactions to virtual character in various virtual reality media such as head-mounted displays.

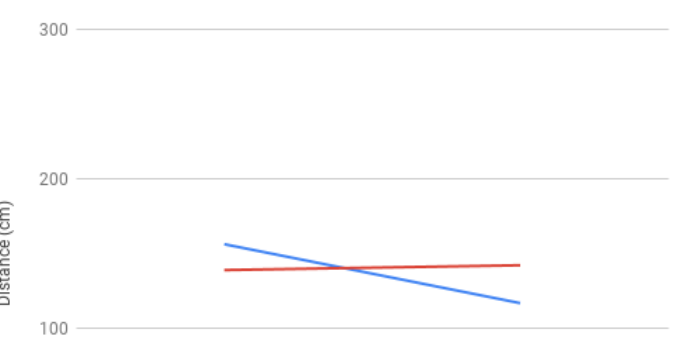

$-\frac{100}{10}$

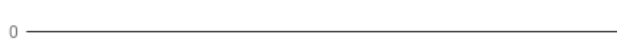

Figure 4: Change in personal distance after 15 minutes playing
One interesting finding is the fall in distance preference in children with ASD after 15 minutes playing with their partner 4. In this setting, it seemed as if the children with ASD initially needed more space between themselves and an unfamiliar partner during the initial phases of interaction, which quickly decreased after a few minutes of engagement. Future research could explore this curve more in the development of activities for social interventions for ASD.

As the children with ASD showed a consistency in need for personal space even when presented with a virtual character, we may assume that proxemics with virtual characters can be taken into consideration when designing virtual environments for social intervention. Ultimately, this work could be further developed to create guidelines which contribute to the development of video game design or avatar-based interaction for autism. Further tests might explore the differences between children with ASD and typically developed children when engaging in free play within virtual environments, and whether social interventions for autism with virtual characters can be more effective when preferences for personal space are implemented.

\section{References}

[1] Association, A. P. Diagnostic and statistical manual of mental disorders (5th ed.), 5 ed. Washington, DC, 2013.

[2] Ballendat, T., Marquardt, N., and Greenberg, S. Proxemic interaction. In ACM International Conference on Interactive Tabletops and Surfaces ITS '10 (2010).

[3] Boyd, L. E., Jiang, X., and Hayes, G. R. ProCom. In Proceedings of the 2017 CHI Conference on Human Factors in Computing Systems - CHI '17 (2017). 
[4] Boyd, L. E., Vikmani, S., and Hayes, G. vrSocial: Toward Immersive Therapeutic VR Systems for Children with Autism Evaluating Effectiveness of Classification Algorithms on Personality Prediction Dataset View project Kawasaki Disease Outbreak Prediction using Neural Networks View project.

[5] Feil-Seifer, D., and Mataric, M. Using proxemics to evaluate human-robot interaction. 5th ACM/IEEE International Conference on Human-Robot Interaction, HRI 2010 (2010).

[6] Gessaroli, E., Santelli, E., di Pellegrino, G., and Frassinetti, F. Personal Space Regulation in Childhood Autism Spectrum Disorders. PLoS ONE (2013).

[7] Hall, E. T. The Hidden Dimension (Anchor Books a Doubleday Anchor Book). 1969.

[8] Hayduk, L. A. Personal space: An evaluative and orienting overview. Psychological Bulletin (1978).

[9] Mora-Guiard, J., Crowell, C., Pares, N., and Heaton, P. Sparking social initiation behaviors in children with
Autism through full-body Interaction. International Journal of Child-Computer Interaction 11 (2017).

[10] Mueller, F., Stellmach, S., Greenberg, S., Dippon, A., Boll, S., Garner, J., Khot, R., Naseem, A., and Altimira, D. Proxemics play. Proceedings of the 2014 conference on Designing interactive systems (2014), 533-542.

[11] Parsons, S., Mitchell, P., \& Leonard, A. The Use and Understanding of Virtual Environments by Adolescents with Autistic Spectrum Disorders. Journal of autism and developmental disorders (2004).

[12] Rogers, A. L., and Fine, H. J. Personal distance in play therapy with an autistic and a symbiotic psychotic child. Psychotherapy: Theory, Research \& Practice (1977).

[13] Welch, K. C., Lahiri, U., Warren, Z., and Sarkar, N. An approach to the design of socially acceptable robots for children with autism spectrum disorders. International Journal of Social Robotics (2010). 